\title{
Size-Dependent Elastic Modulus and Vibration Frequency of Nanocrystals
}

\author{
Lihong Liang, Hansong Ma, and Yueguang Wei \\ LNM, Institute of Mechanics, Chinese Academy of Sciences, Beijing 100190, China \\ Correspondence should be addressed to Lihong Liang, lianglh@lnm.imech.ac.cn
}

Received 27 May 2010; Revised 1 July 2010; Accepted 7 July 2010

Academic Editor: Quanqin Dai

Copyright () 2011 Lihong Liang et al. This is an open access article distributed under the Creative Commons Attribution License, which permits unrestricted use, distribution, and reproduction in any medium, provided the original work is properly cited.

The elastic properties and the vibration characterization are important for the stability of materials and devices, especially for nanomaterials with potential and broad application. Nanomaterials show different properties from the corresponding bulk materials; the valid theoretical model about the size effect of the elastic modulus and the vibration frequency is significant to guide the application of nanomaterials. In this paper, a unified analytical model about the size-dependent elastic modulus and vibration frequency of nanocrystalline metals, ceramics and semiconductors is established based on the inherent lattice strain and the binding energy change of nanocrystals compared with the bulk crystals, and the intrinsic correlation between the elasticity and the vibration properties is discussed. The theoretical predictions for $\mathrm{Cu}, \mathrm{Ag}$, $\mathrm{Si}$ thin films, nanoparticles, and $\mathrm{TiO}_{2}$ nanoparticles agree with the experimental results, the computational simulations, and the other theoretical models.

\section{Introduction}

Nanomaterials, including nanoparticles, nanowires, nanotubes, and nanoscale thin films, have been found to show different physical, chemical, and mechanical properties from the corresponding bulk materials [1-16], such as the phonon frequency blue shift of nano-semiconductors and nanometals [2-7], the elastic modulus increase of thin films and nanoparticles [8-14], the melting temperature, and the thermal conductivity decrease of nanocrystals $[15,16]$; these peculiar properties bring potential and broad application in microelectronics, optics, sensor, and so forth. While the elasticity and the vibration characterization of nanomaterials directly determines the stability and the reliability of the devices; therefore, to understand the size effect of elasticity and vibration properties and their theoretical mechanism is important. The theoretical explanations for the size effect of the elastic modulus are related with the surface effect by introducing the surface energy contribution in the continuum mechanics [11] or by the computational simulations reflecting the surface stress $[8,9]$ or surface relaxation influence $[13,17]$. The size effect of the phonon frequency is attributed to the phonon confinement [3], the surface pressure [18], or the interfacial vibration effects [19], and so forth, Although various theoretical interpretations were, respectively, proposed for the elastic modulus and the phonon frequency change of nanomaterials [2-6, 8$11,13,14,17-19]$, a unified theory about the size-dependent elastic modulus and phonon frequency, with all parameters having clear physical meaning, is lacking, which is helpful to understand the physical mechanism and the inherent correlation of the elasticity and the vibration properties, and helpful to guide the application of nanomaterials.

It is known that the Young's modulus $Y$ and the phonon vibration frequency $v$ are both related with the force constant $k[20,21]$

$$
Y=\frac{k}{h}, \quad v=C K^{1 / 2},
$$

where $h$ is the atomic distance in equilibrium or the bond length, and $C$ is a constant. The force constant $k=$ $d^{2} u(r) / d r_{(r=h)}^{2}$, where $u(r)$ denotes the interatomic potential, which is a function of the atomic distance $r$; therefore, the Young's modulus and the vibration frequency are both inherently related with the atomic interaction energy and the atomic distance. Recently, an analytic thermodynamic equation for the surface stress and the size-dependent lattice strain of nanocrystals has been established [22]. In terms 
of this equation, the bond length change of nanocrystals compared with the bulk crystals can be determined. Thus, it is also possible to develop an analytic thermodynamic equation for the size-dependent elastic modulus and vibration frequency combining with the consideration of the binding energy change, which will be useful for the estimation of elasticity and the vibration properties of nanomaterials and for understanding the effect of the thermodynamic parameters on the elasticity and the vibration properties.

In this paper, a quantitative unified model without any free parameter for the size-dependent elastic modulus and vibration frequency of single crystal thin films and nanocrystals with grain boundaries is established based on the size-dependent bond length and bond energy. The predictions of the model for the enhancement of the elastic modulus and the vibration frequency of $\mathrm{Cu}, \mathrm{Ag}$, Si thin films, nanoparticles, and $\mathrm{TiO}_{2}$ nanoparticles agree with the results of the molecular dynamics (MD) simulations, the continuum mechanics calculations, and the experimental measurements of different authors.

\section{Model}

According to the theory of the solid state physics, the interatomic potential of an ideal crystal can be expressed as $u(r)=[p q /(p-q)] e\left[(h / r)^{p / p}-(h / r)^{q} / q\right]$, where $e$ is the atomic binding energy or the bond energy, and the coefficients $p$ and $q$ depend on the shapes of the potential curves (when $p=12$ and $q=6$, the potential is the Lennard-Jones (L-J) potential) [33]. Therefore, $k=d^{2} u(r) / d r_{(r=h)}^{2}=p q e / h^{2}$ and combining with (1),

$$
Y=\frac{C^{\prime} e}{h^{3}}, \quad v=\frac{C^{\prime \prime} e^{1 / 2}}{h},
$$

where $C^{\prime}=p q$ and $C^{\prime \prime}=C(p q)^{1 / 2}$ are constants for a crystal, that is, the elastic modulus and the phonon vibration frequency are both dependent on the bond length $h$ and the bond energy $e$ of the crystal. Note that for metals, ceramics and semiconductors, the EAM (embedded-atom method) potential, the Morse potential, and the Tersoff potential are more appropriate, respectively, but the intrinsic relations among the force constant, the elastic modulus, the frequency, the bond energy, and the bond length are the same as those in the simple L-J potential as shown in (2). Let $Y(D)$ and $v(D)$ denote the size-dependent Young's modulus and vibration frequency of nanomaterials, where $D$ is the thickness of single crystal thin films or the diameter of nanoparticles or the grains, assuming that (2) is still applicable to nanomaterials, $Y(D)=C^{\prime} e(D) / h(D)^{3}, v(D)=C^{\prime \prime} e(D)^{1 / 2} / h(D)$, where $e(D)$ and $h(D)$ are the corresponding size-dependent average bond energy and bond length of nanomaterials, respectively. Then the ratio $Y(D) / Y$ and $v(D) / v$ can be written as

$$
\frac{Y(D)}{Y}=\left[\frac{h}{h(D)}\right]^{3}\left[\frac{e(D)}{e}\right], \quad \frac{v(D)}{v}=\left[\frac{h}{h(D)}\right]\left[\frac{e(D)}{e}\right]^{1 / 2} \text {. }
$$

In (3), the size-dependent bond length $h / h(D)$ is related with the inherent lattice strain $\varepsilon=[h(D)-h] / h$ of nanocrystals. According to the Laplace-Young equation [34], the hydrostatic pressure acting on a small solid sphere particle immersed in the liquid $P=4 \sigma / D$, with the isotropic surface stress $\sigma$ and the diameter $D$, will induce an elastic strain $\varepsilon$ in the particle. Under the small strain, $\varepsilon=\triangle D / D$ $=\Delta A /(2 A)=\Delta V /(3 V)$ with the area $A$ and the volume $V$ of the particle. Combining with the definition of the compressibility $\kappa=-\triangle V /(V P), P=-3 B \varepsilon$, where $B=1 / \kappa$ is the bulk modulus; therefore, $\varepsilon=-4 \kappa \sigma /(3 D)$. According to the thermodynamic definition of the surface stress [22], $\sigma=\partial G / \partial A=\partial(\gamma A) / \partial A=\gamma+A \partial \gamma / \partial A \approx \gamma+A \Delta \gamma / \Delta A$, where $G$ is the surface/interface excess Gibbs free energy, $\gamma$ is the surface/interface energy, $\triangle A / A=-8 \kappa \sigma /(3 D)$, $\Delta \gamma=\gamma-\gamma_{b}$ with the bulk surface/interface energy $\gamma_{b}$, thus, $\gamma=\gamma_{b}-(\sigma-\gamma) 8 \kappa \sigma /(3 D)$. Considering the size dependence of the surface/interface energy, $\gamma=\gamma_{b}\left(1-D_{0} / D\right)$, where $D_{0}$ is a minimum critical size, and $D_{0}=2 h, 3 h$ for thin films and particles, respectively [22]. Therefore, the intrinsic surface stress $\sigma= \pm\left[\left(3 \gamma_{b} D_{0}\right) /(8 \kappa)\right]^{1 / 2}$, where the positive sign denotes the tension stress, the negative denotes the compression stress, $\gamma_{b}=2 h S_{v} H_{m} /\left(3 V_{c} R\right)$ is the bulk solid-liquid interface energy [23], $S_{v}$ denotes the vibrational part of the melting entropy $S_{m}, H_{m}$ is the melting enthalpy, $V_{c}$ is the molar volume of crystals, and $R$ is the ideal gas constant. Thus, $\varepsilon= \pm(2 / 3 D) \sqrt{\kappa D_{0} h S_{v} H_{m} /\left(V_{c} R\right)}$ for free-standing single crystal particles and thin films, similarly, $\varepsilon=-2 \kappa \sigma /(3 D)= \pm(1 / 3 D) \sqrt{\kappa D_{0} h S_{v} H_{m} /\left(V_{c} R\right)}$ for nanocrystalline materials with grain boundaries considering the smaller strain of solid-solid interfaces [23]. Therefore,

$$
\frac{h}{h(D)}=\frac{1}{1 \pm(f / 3 D) \sqrt{\kappa D_{0} h S_{v} H_{m} /\left(V_{c} R\right)}}
$$

where $f=1,2$ for nanocrystals with grain boundaries and single crystal thin films, respectively, the negative denotes the lattice contraction, and the positive denotes the lattice expansion. Note that $\kappa$ in (4) is assumed to be sizeindependent as the first-order approximation, which does not lead to a big error on the $Y(D) / Y$ value in $(3)$ since $\varepsilon \ll 1[22]$. According to (4), the $D$-dependent average bond length of nanocrystals can be obtained by some available thermodynamic parameters such as the vibration entropy and the melting enthalpy.

The size dependence of the average bond energy is related with the size effects of the cohesive energy of crystals and the coordination number of atoms. Let the coordination number of an atom in the bulk crystals be $Z$, and the corresponding cohesive energy of one molar single crystal $E=\left(Z N_{0}\right) e / 2$ for the bulk materials, where $N_{0}$ is Avogadro constant, that is, $e=2 E /\left(Z N_{0}\right)$. For thin films and nanoparticles, the size-dependent cohesive energy $E(D)=$ $[(Z-m) y+Z(1-y)] N_{0} e(D) / 2$ considering the larger surfacevolume-ratio and the surfacial breaking bond effect, where $y=D_{0} / D$ represents the ratio of the surface atoms number to the volume atoms number, and $m$ denotes the decrease of the surfacial atomic coordination number compared with $Z$, that is, $e(D)=2 E(D) /\left[(Z-m y) N_{0}\right]$. Therefore, $e(D) / e=$ $[E(D) / E] /[1-y m / Z]$. Approximately, $E-E(D)=\gamma_{0} A$, where $\gamma_{0}$ is the surface or solid-solid interface energy for freestanding thin films, or nanocrystals with grain boundaries, 


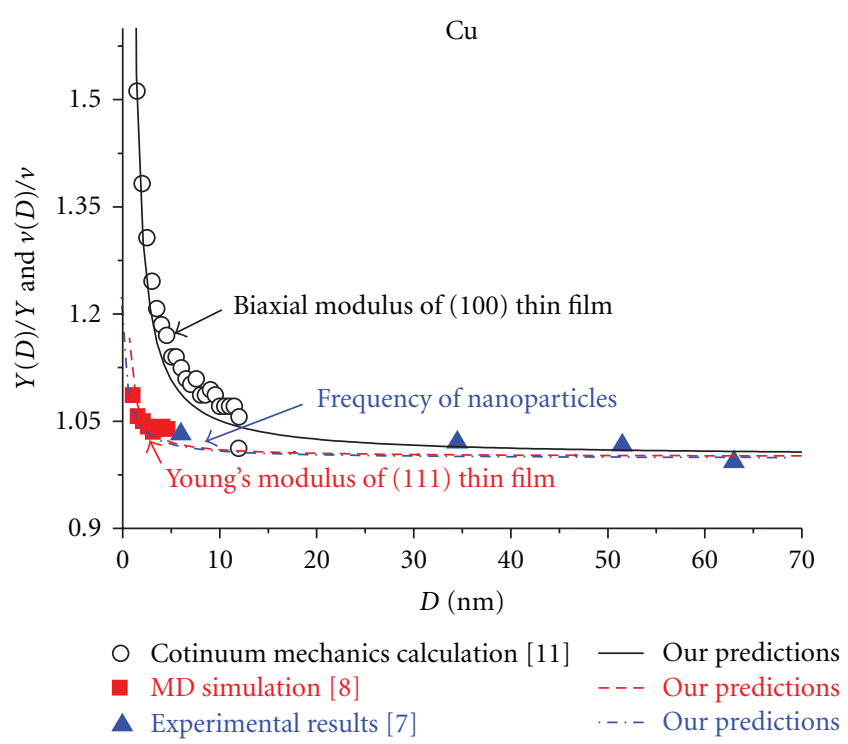

Figure 1: Size-dependent elastic modulus and vibration frequency of $\mathrm{Cu}$ thin films and nanocrystals. In (6), $S_{v} \approx S_{m}=H_{m} / T_{m}=$ $9.613 \mathrm{Jmol}^{-1} \mathrm{~K}^{-1}$ for metals [23], $T_{m}$ is the melting temperature. $m=3,4$, respectively, for (111) and (100) faces corresponding to the computational simulation [8] and continuum mechanics calculation [11] of thin films. For nanocrystalline $\mathrm{Cu}$, the solid-solid interface energy $\gamma_{0} \approx 2 \gamma_{b}=4 h S_{v} H_{m} /\left(3 V_{c} R\right)=0.724 \mathrm{Jm}^{-2}$ [23], for $\mathrm{Cu}$ thin films, $\gamma_{0}$ is the surface energy. Other related parameters are in Table 1.

and $A$ is the surface/interface area, $A=(2 / D) V_{c}$ for thin films and $A=(3 / D) V_{c}$ for nanocrystals with grain boundaries. Thus, we have

$$
\frac{e(D)}{e}=\frac{1-\gamma_{0} A / E}{1-\left(D_{0} / D\right)(m / Z)} .
$$

According to (5), the $D$-dependent average bond energy of nanocrystals can be obtained by the available thermodynamic parameters such as the cohesive energy and the surface/interface energy. Finally, substituting (4) and (5) into (3), the size-dependent elastic modulus and vibration frequency can be determined as follows:

$$
\begin{aligned}
\frac{Y(D)}{Y}= & {\left[\frac{1}{1 \pm f / 3 D \sqrt{\kappa D_{0} h S_{v} H_{m} /\left(V_{c} R\right)}}\right]^{3} \frac{1-\gamma_{0} A / E}{1-\left(D_{0} / D\right)(m / Z)}, } \\
& \frac{v(D)}{v}=\left[\frac{Y(D)}{Y}\right]^{1 / 3}\left[\frac{1-\gamma_{0} A / E}{1-\left(D_{0} / D\right)(m / Z)}\right]^{3 / 2} .
\end{aligned}
$$

\section{Results and Discussion}

Figure 1 shows the thickness-dependent Young' modulus and biaxial modulus of $\mathrm{Cu}$ thin films and the diameterdependent vibration frequency of $\mathrm{Cu}$ nanoparticles. Figure 2 shows the grain diameter-dependent bulk modulus and vibration frequency of $\mathrm{TiO}_{2}$ nanocrystals. Figure 3 shows the thickness-dependent biaxial modulus of $\mathrm{Ag}$ thin films and

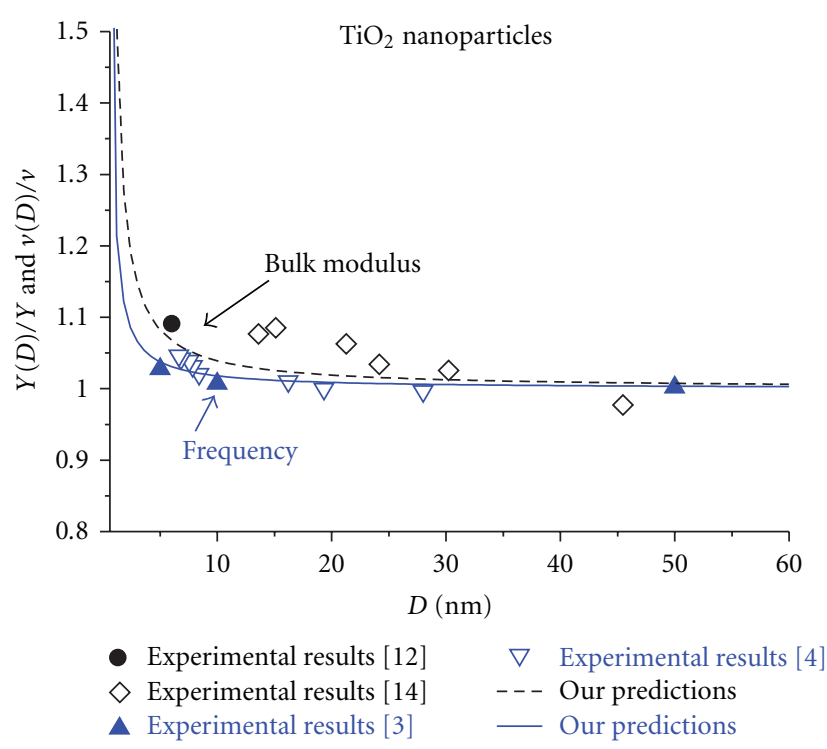

FIGURE 2: Size-dependent elastic modulus and vibration frequency of $\mathrm{TiO}_{2}$ nanocrystals. In (6), $h=a / \sqrt{2}$ with the lattice constant $a=0.458 \mathrm{~nm}[24] . S_{v} \approx 0.4 S_{m}=0.4 H_{m} / T_{m}=12.576 \mathrm{Jmol}^{-1} \mathrm{~K}^{-1}$ for semiconductors [25]. $V_{c}=M / \rho$ with the molar mass $M=$ $79.88 \mathrm{~g} \mathrm{~mol}^{-1}$ and the density $\rho=3.84 \mathrm{~g} \mathrm{~cm}^{-3}[24], m=7, \gamma_{0} \approx$ $2 \gamma_{b}$. Other parameters are in Table 1 .

the diameter-dependent vibration frequency of Ag nanoparticles. Figure 4 shows the thickness-dependent Young' modulus of Si thin films and the diameter-dependent vibration frequency of Si nanocrystals. The lines are our theoretical predictions based on (6) and some available thermodynamic parameters [24-32]; the symbols are the results of the MD simulations $[8,9]$, the continuum mechanics and the semicontinuum model calculations $[11,13]$, the phonon dispersion calculation [5], and the experimental measurements $[2-4,7,12-14]$. The agreement between our model's predictions and the experimental results, and the simulations and the calculations of other groups can be found from the figures. It can be seen that the elastic modulus of nanomaterials in the size range of $1-50 \mathrm{~nm}$ increases about $1 \%-50 \%$, and the frequency of nanomaterials in the size range of $1-70 \mathrm{~nm}$ increases about $1 \%-30 \%$; especially the size effects of the modulus and the frequency are obvious and should be considered in the size of smaller than about $20 \mathrm{~nm}$ and $10 \mathrm{~nm}$, respectively, due to the larger change of the bond length and the bond energy in the smaller scale. The enhancement of the frequency is smaller than that of the elastic modulus for the same nanosystems, which is reasonable since $Y(D) / Y=[v(D) / v]^{2}[h / h(D)]$ based on (3) and the background of the lattice contraction, that is, $h / h(D)>1$ (The negative is taken in (6)). The lattice constants of $\mathrm{TiO}_{2}$ nanoparticles [35] and some metallic nanocrystals [22] have been observed to decrease compared with the corresponding bulk crystals. The above relation is also in agreement with the general knowledge of $Y \propto v^{2}$, which implies that the elasticity change is originated from the lattice vibration change of nanocrystals. 
TABLE 1: The related parameters in (4)-(6).

\begin{tabular}{|c|c|c|c|c|c|c|c|c|}
\hline & $h(\mathrm{~nm})$ & $H_{m}\left(\mathrm{~K} \mathrm{Jmol}^{-1}\right)$ & $T_{m}(\mathrm{~K})$ & $B(\mathrm{GPa})$ & $V_{c}\left(\mathrm{~cm}^{3} \mathrm{~mol}^{-1}\right)$ & $\gamma_{0}\left(\mathrm{Jm}^{-2}\right)$ & $E\left(\mathrm{KJ} \mathrm{mol}^{-1}\right)$ & $Z$ \\
\hline $\mathrm{Cu}$ & $0.2556[26]$ & $13.05[27]$ & 1357.6 [27] & $137.8[28]$ & $7.1[27]$ & $1.952[29]$ & $336[30]$ & 12 \\
\hline $\mathrm{TiO}_{2}$ & 0.3239 & $66.88[24]$ & 2128 [24] & $220[31]$ & 20.802 & 2.1 & $672.4[24]$ & 14 \\
\hline $\mathrm{Ag}$ & $0.2194[26]$ & 11.3 [27] & 1234 [27] & $103.6[28]$ & 10.3 [27] & 1.2 [29] & $284[30]$ & 12 \\
\hline Si & $0.3368[26]$ & 50.55 [27] & 1685 [27] & $235.4[28]$ & $12.1[27]$ & $1.568[32]$ & $446[30]$ & 16 \\
\hline
\end{tabular}

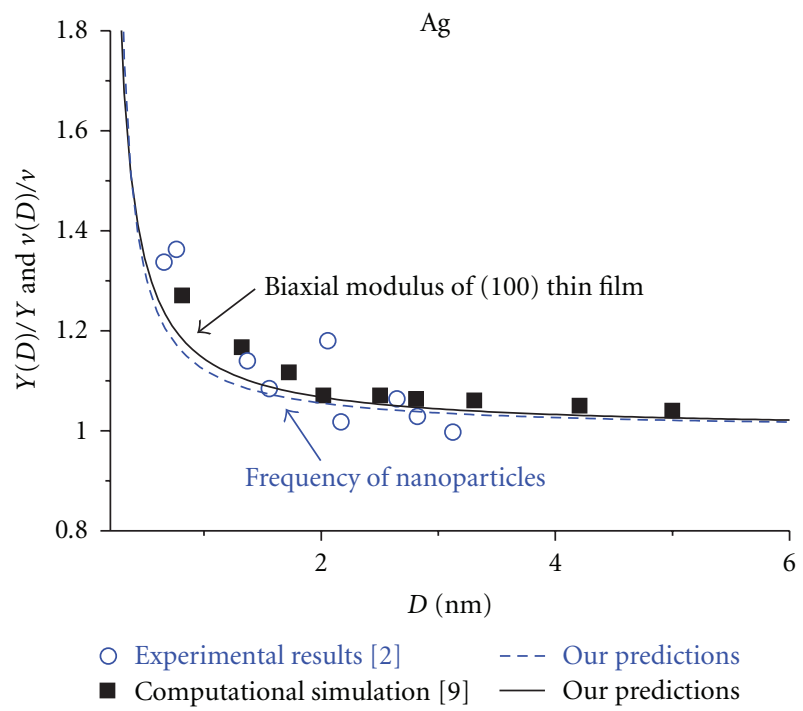

FIGURE 3: Size-dependent elastic modulus and vibration frequency of $\mathrm{Ag}$ thin film and nanocrystals. In (6), $S_{v} \approx H_{m} / T_{m}=$ 9.157 $\mathrm{Jmol}^{-1} \mathrm{~K}^{-1}, m=4$. For nanocrystalline Ag, $\gamma_{0} \approx 2 \gamma_{b}=$ $0.4 \mathrm{Jm}^{-2}$, for Ag thin films, $\gamma_{0}$ is the surface energy. Other related parameters are in Table 1.

According to (3), when the average bond length contracts and the bond energy increases with reducing size of nanocrystals, the elastic modulus and the vibration frequency enhance. The model indicates that the modulus enhancement and the phonon frequency blue shift originate from several contributions: one is the intrinsic surface tension stress and the average lattice contraction of nanocrystals determined by (4), which causes the change of the lattice vibration and the elastic properties compared with the corresponding bulk counterparts; at the same time, the average atomic binding strengthening, resulted from the intrinsic small size effect (the cohesive energy change) and the surface breaking bonds and the large surface ratio based on (5), contributes to the elastic and vibration behavior change. In fact, the physics of our model in some degree conforms to the theory of the surface bond contraction and the bond strengthening $[21,36]$. On the other hand, our model implies not only the surface effect but also the internal contribution, for example, the bond length contraction and the bond energy increase of the interior atoms originated from the phonon confinement effect have both influence on the elasticity and the vibration behavior of nanocrystals, which is nonlinearly dependent on $1 / D$ different from the previous discussion [37].

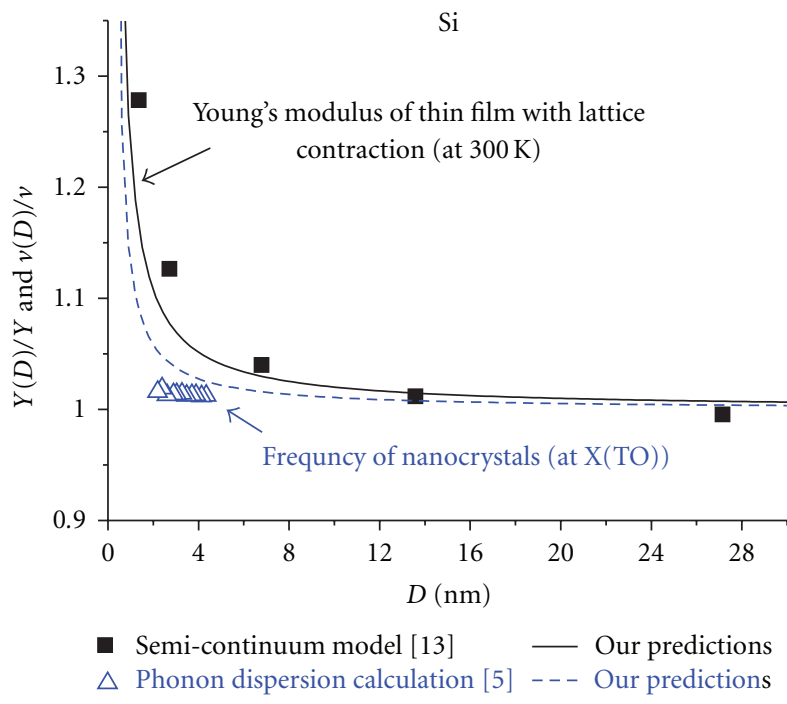

FIGURE 4: Size-dependent elastic modulus and vibration frequency of Si thin film and nanocrystals. In (6), $S_{v}=6.72 \mathrm{Jmol}^{-1} \mathrm{~K}^{-1}[25]$, $m=6$. For nanocrystalline Si, $\gamma_{0} \approx 2 \gamma_{b}=1.06 \mathrm{Jm}^{-2}$, for $\mathrm{Si}$ thin films, $\gamma_{0}$ is the surface energy. Other related parameters are in Table 1 .

Note that the model prediction is based on the isotropic assumption for nanoparticles. When the change trends of the bond length and the bond energy are different in different orientations, the elasticity and the vibration behavior will exhibit diversity. For example, the modulus decrease and the frequency red shift were observed for some nanosolids $[18,38]$, which may be because of the bond length expansion and the bond energy decrease. According to (3), even if the bond length expands, as long as the bond energy enhances enough to compensate for it, or the bond energy decreases but the bond length contracts enough, the modulus increase and the frequency blue shift may occur, vice versa. In that case, the surface stress and the lattice strain state, the surface atomic coordinate number and the surface/interface energy will be different.

\section{Conclusion}

In conclusion, the elastic modulus and the vibration frequency of metal, ceramic, and semiconductor nanocrystals are dependent on the thickness of thin films and the diameter of nanoparticles. This size effect is modeled by considering the size-dependent bond length and bond energy related with the surface effect and the interior contribution. 
The model is expressed by some available thermodynamic parameters, and the predictions for $\mathrm{Cu}, \mathrm{Ag}, \mathrm{Si}$ thin films, nanoparticles, and $\mathrm{TiO}_{2}$ nanoparticles are in agreement with the computational simulations, the continuum mechanics calculations, and the experimental results.

\section{Acknowledgment}

The financial supports from the NSFC under Grants no. 10802088, 10832008, 10702070, 10932011 and The LNM initial funding for young investigators are acknowledged.

\section{References}

[1] H. Gleiter, "Nanostructured materials: basic concepts and microstructure," Acta Materialia, vol. 48, no. 1, pp. 1-29, 2000.

[2] M. Fujii, T. Nagareda, S. Hayashi, and K. Yamamoto, "Lowfrequency Raman scattering from small silver particles embedded in $\mathrm{SiO}_{2}$ thin films," Physical Review B, vol. 44, no. 12, pp. 6243-6248, 1991.

[3] D. Bersani, P. P. Lottici, and X.-Z. Ding, "Phonon confinement effects in the Raman scattering by $\mathrm{TiO}_{2}$ nanocrystals," Applied Physics Letters, vol. 72, no. 1, pp. 73-75, 1998.

[4] W. F. Zhang, Y. He, M. S. Zhang, Z. Yin, and Q. Chen, "Raman scattering study on anatase $\mathrm{TiO}_{2}$ nanocrystals," Journal of Physics D, vol. 33, no. 8, pp. 912-916, 2000.

[5] X. Hu and J. Zi, "Reconstruction of phonon dispersion in $\mathrm{Si}$ nanocrystals," Journal of Physics: Condensed Matter, vol. 14, no. 41, pp. L671-L677, 2002.

[6] L.-H. Liang, C.-M. Shen, X.-P. Chen, W.-M. Liu, and H.-J. Gao, "The size-dependent phonon frequency of semiconductor nanocrystals," Journal of Physics: Condensed Matter, vol. 16, no. 3, pp. 267-272, 2004.

[7] O. A. Yeshchenko, I. M. Dmitruk, A. M. Dmytruk, and A. A. Alexeenko, "Influence of annealing conditions on size and optical properties of copper nanoparticles embedded in silica matrix," Materials Science and Engineering B, vol. 137, no. 1-3, pp. 247-254, 2007.

[8] F. H. Streitz, K. Sieradzki, and R. C. Cammarata, "Elastic properties of thin fcc films," Physical Review B, vol. 41, no. 17, pp. 12285-12287, 1990.

[9] F. H. Streitz, R. C. Cammarata, and K. Sieradzki, "Surfacestress effects on elastic properties. I. Thin metal films," Physical Review B, vol. 49, no. 15, pp. 10699-10706, 1994.

[10] L. H. Liang, J. C. Li, and Q. Jiang, "Size-dependent elastic modulus of $\mathrm{Cu}$ and $\mathrm{Au}$ thin films," Solid State Communications, vol. 121, no. 8, pp. 453-455, 2002.

[11] R. Dingreville, J. M. Qu, and M. Cherkaoui, "Surface free energy and its effect on the elastic behavior of nano-sized particles, wires and films," Journal of the Mechanics and Physics of Solids, vol. 53, no. 8, pp. 1827-1854, 2005.

[12] V. Pischedda, G. R. Hearne, A. M. Dawe, and J. E. Lowther, "Ultrastability and enhanced stiffness of $\sim 6 \mathrm{~nm} \mathrm{TiO}_{2}$ nanoanatase and eventual pressure-induced disorder on the nanometer scale," Physical Review Letters, vol. 96, no. 3, Article ID 035509, 4 pages, 2006.

[13] J.-H. Zhang, Q.-A. Huang, H. Yu, and J. Wang, "The influence of surface effects on size-dependent mechanical properties of silicon nanobeams at finite temperature," Journal of Physics D, vol. 42, no. 4, Article ID 045409, 6 pages, 2009.

[14] B. Chen, H. Zhang, K. A. Dunphy-Guzman et al., "Sizedependent elasticity of nanocrystalline titania," Physical Review B, vol. 79, no. 12, Article ID 125406, 8 pages, 2009.
[15] Q. Jiang, H. X. Shi, and M. Zhao, "Melting thermodynamics of organic nanocrystals," Journal of Chemical Physics, vol. 111, no. 5, pp. 2176-2180, 1999.

[16] L. H. Liang and B. Li, "Size-dependent thermal conductivity of nanoscale semiconducting systems," Physical Review B, vol. 73, no. 15, Article ID 153303, 4 pages, 2006.

[17] J.-G. Guo and Y.-P. Zhao, "The size-dependent elastic properties of nanofilms with surface effects," Journal of Applied Physics, vol. 98, no. 7, Article ID 074306, 11 pages, 2005.

[18] J. Meng, G. Zou, Q. Ciu, Y. Zhao, and Z. Zhu, "Raman scattering from $\mathrm{PbTiO}_{3}$ of various grain sizes at high hydrostatic pressures," Journal of Physics: Condensed Matter, vol. 6, no. 32, pp. 6543-6548, 1994.

[19] X. L. Wu, G. G. Siu, S. Tong, et al., "Raman scattering of alternating nanocrystalline silicon/amorphous silicon multilayers," Applied Physics Letters, vol. 69, no. 4, pp. 523-525, 1996.

[20] W. D. Nix and H. Gao, "An atomistic interpretation of interface stress," Scripta Materialia, vol. 39, no. 12, pp. 16531661, 1998.

[21] C. Q. Sun, B. K. Tay, X. T. Zeng et al., "Bond-order-bondlength-bond-strength (bond-OLS) correlation mechanism for the shape-and-size dependence of a nanosolid," Journal of Physics: Condensed Matter, vol. 14, no. 34, pp. 7781-7795, 2002.

[22] Q. Jiang, L. H. Liang, and D. S. Zhao, "Lattice contraction and surface stress of fcc nanocrystals," Journal of Physical Chemistry B, vol. 105, no. 27, pp. 6275-6277, 2001.

[23] Q. Jiang, D. S. Zhao, and M. Zhao, "Size-dependent interface energy and related interface stress," Acta Materialia, vol. 49, no. 16, pp. 3143-3147, 2001.

[24] R. C. Weast, Handbook of Chemistry and Physics, Chemical Rubber, Cleveland, Ohio, USA, 70th edition, 1989-1990.

[25] A. R. Regel' and V. M. Glazov, "Entropy of melting of semiconductors," Semiconductors, vol. 29, no. 5, p. 405, 1995.

[26] H. W. King, in Physical Metallurgy, R. W. Cahn, Ed., p. 60, North-Holland, Amsterdam, The Netherlands, 1970.

[27] Table of Periodic Properties of the Elements, Sargent-Welch Scientific, Skokie, Ill, USA, 1980.

[28] E. A. Brandes, Ed., Smithells Metals Reference Book, Butterworth, London, UK, 6th edition, 1983.

[29] L. Vitos, A. V. Ruban, H. L. Skriver, and J. Kollár, "The surface energy of metals," Surface Science, vol. 411, no. 1-2, pp. 186202, 1998.

[30] C. Kittel, Introduction to Solid State Physics, John Wiley \& Sons, Chichester, UK, 5th edition, 1976.

[31] V. Swamy, E. Holbig, L. S. Dubrovinsky, V. Prakapenka, and B. C. Muddle, "Mechanical properties of bulk and nanoscale $\mathrm{TiO}_{2}$ phases," Journal of Physics and Chemistry of Solids, vol. 69, no. 9, pp. 2332-2335, 2008.

[32] D. M. Bird, L. J. Clarke, R. D. King-Smith, M. C. Payne, I. Stich, and A. P. Sutton, "First principles calculation of the structure and energy of Si(113)," Physical Review Letters, vol. 69, no. 26, pp. 3785-3788, 1992.

[33] J.-G. Guo, L.-J. Zhou, and Y.-P. Zhao, "Size-dependent elastic modulus and fracture toughness of the nanofilm with surface effects," Surface Review and Letters, vol. 15, no. 5, pp. 599-603, 2008.

[34] R. C. Cammarata and K. Sieradzki, "Surface and interface stresses," Annual Review of Materials Science, vol. 24, no. 1, pp. 215-234, 1994.

[35] V. Swamy, L. S. Dubrovinsky, N. A. Dubrovinskaia et al., "Compression behavior of nanocrystalline anatase $\mathrm{TiO}_{2}$," Solid State Communications, vol. 125, no. 2, pp. 111-115, 2003. 
[36] M. Gu, Y. Zhou, L. Pan, Z. Sun, S. Wang, and C. Q. Sun, "Temperature dependence of the elastic and vibronic behavior of Si, Ge, and diamond crystals," Journal of Applied Physics, vol. 102, no. 8, Article ID 083524, 4 pages, 2007.

[37] M. Fujii, Y. Kanzawa, S. Hayashi, and K. Yamamoto, "Raman scattering from acoustic phonons confined in Si nanocrystals," Physical Review B, vol. 54, no. 12, pp. R8373-R8376, 1996.

[38] X. Y. Qin, X. R. Zhang, G. S. Cheng, and L. D. Zhang, "The elastic properties of nanostructured $\mathrm{Ag}$ measured by laser ultrasonic technique," Nanostructured Materials, vol. 10, no. 4, pp. 661-672, 1998. 

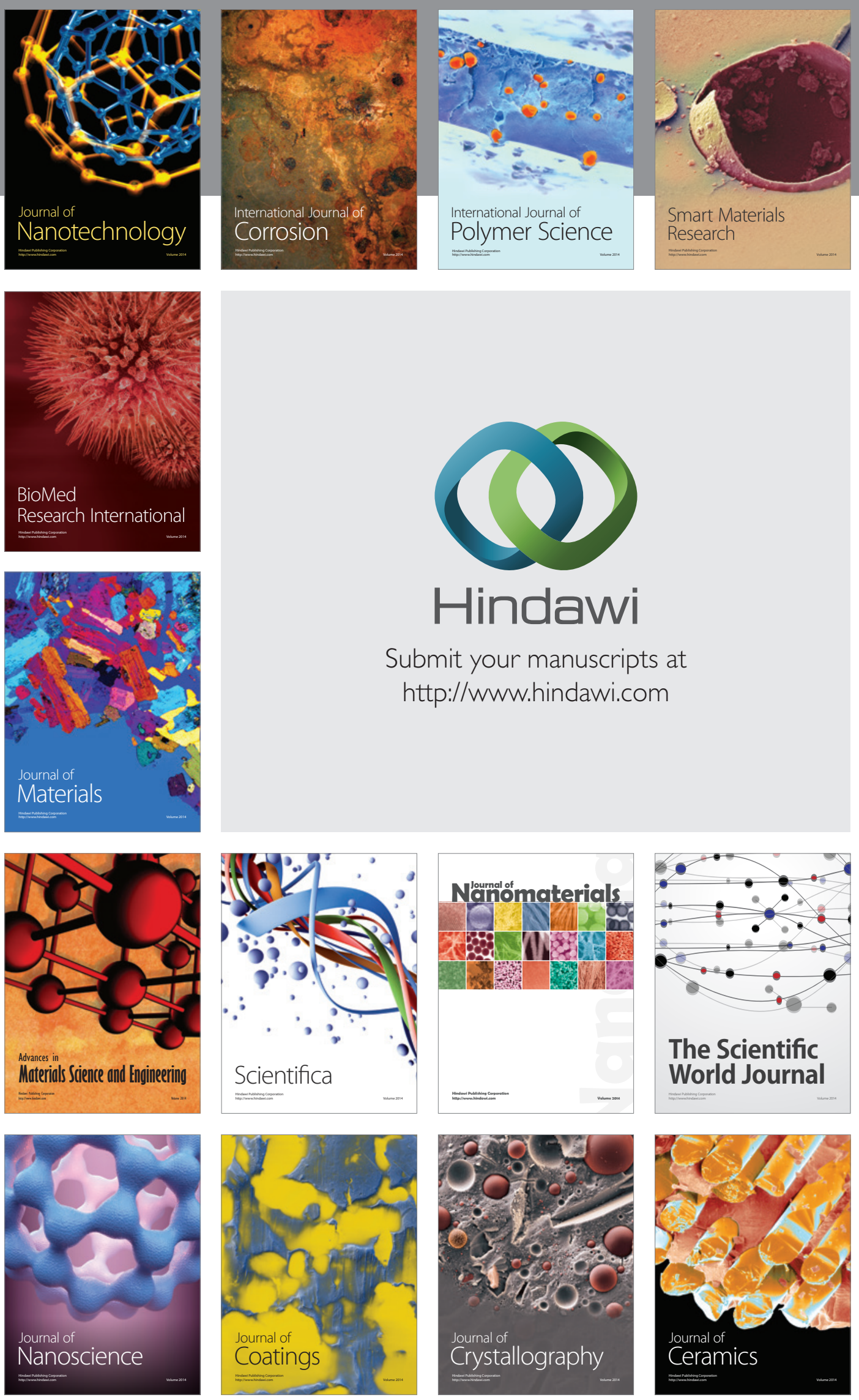

The Scientific World Journal

Submit your manuscripts at

http://www.hindawi.com

\section{World Journal}

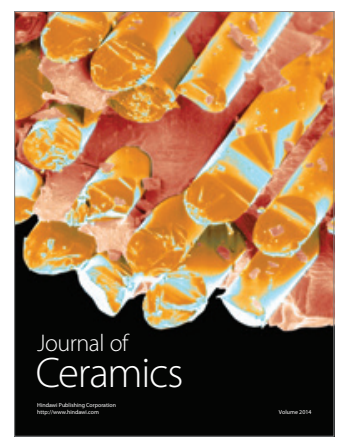

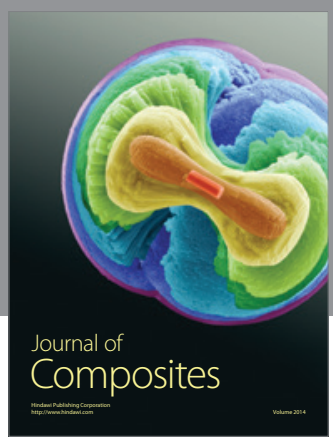
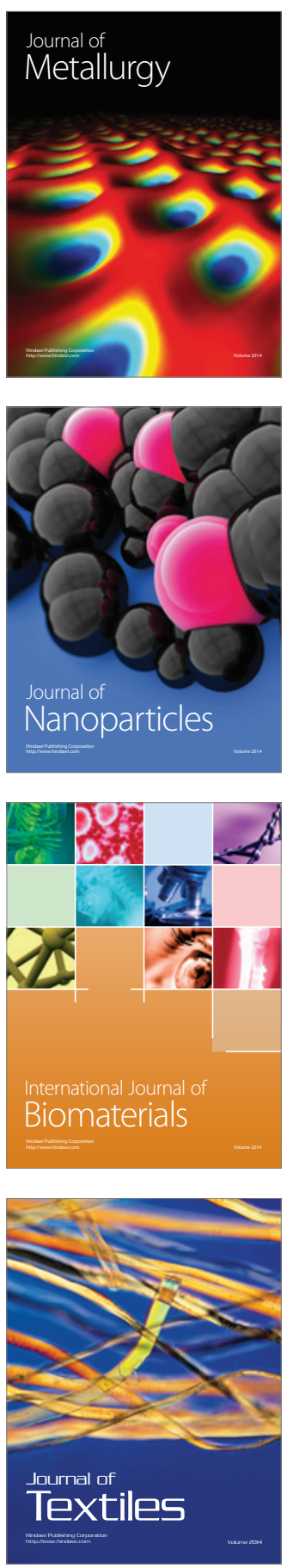